Results Evaluation of outcomes of gestation showed that in 9 cases, there has been a termination of pregnancy in the $1 \mathrm{st}$ trimester due to the high activity of SLE. They were mostly women with active lupus nephritis (7), one case with massive exudative pericarditis and one patient with lupus hepatitis. Pregnancy in 6 women on the background of a moderate (2) and minimal disease activity (4), with a primary skin (4) and articular syndrome (1), with the trace proteinuria (1) completed delivery of healthy children in the gestation of 36 to 38 weeks. In 3 of them - by Caesarean section, in 3 others it was delivery was vaginally at 39 weeks gestation. 27 did not have children because of the risk of adverse effects of pregnancy on the course of the disease; the other 27 women had children prior to the development of SLE.

Conclusions Half of the women (52\%) was not able to have children due to the high risk of adverse effects of pregnancy on the course of the disease, with active SLE caused the interruption of pregnancy in $13 \%$ of patients.

\section{PREGNANCY OUTCOME OF SYSTEMIC LUPUS ERYTHEMATOSUS PATIENTS IN CIPTO MANGUNKUSUMO NATIONAL REFERRAL HOSPITAL, JAKARTA, INDONESIA}

SA Kusumo Wibowo*, A Ariane, SP Hidayat, EJ Yunasan, B Setiyohadi, R Hidayat, S Sarmidi, Z Albar, H Isbagio. RSCM, Division of Rheumatology- Department of Internal Medicine, Central Jakarta, Indonesia

\subsection{6/lupus-2017-000215.391}

Background and aims Systemic Lupus Erythematosus (SLE) is a multisystem autoimmune disease which commonly affects women of childbearing age. There were reports of the adverse pregnancy outcome in SLE patients but data from Indonesia was still lacking. The objectives of this study were to analyse the outcome of pregnancy in SLE patients and to identify the factors associated with pregnancy outcome.

Methods This was a retrospective study of pregnant SLE patients in Indonesian national referral hospital from 20122015. Medical records of all pregnant SLE patients who gave birth in our centre during that period were reviewed. Independent variables were previous and current nephritis, history of adverse pregnancy outcome, pre-pregnancy hypertension, and disease control during pregnancy. Dependent variables were maternal complications (ICU admission, SLE flare, hypertension/eclampsia/preeclampsia, and death) and fetal/neonatal complications (low birth weight, oligohydramnios, and abortion/fetal death/neonatal death).

Results There were 32 pregnancies of which 13 were first pregnancies. Preterm deliveries were observed in $37.5 \%$ patients and $66.7 \%$ patients were delivered by C-section. Eight patients (25\%) experienced maternal complications with 1 patient died due to heart failure. There were $71.9 \%$ pregnancies with fetal/neonatal complications and among those, low birth weight was the main complication (39.1\%). There were significant association between disease control during pregnancy and maternal complications $(\mathrm{p}=0.029)$ and between history of adverse pregnancy outcome and fetal/neonatal complications $(p=0.005)$.

Conclusions The rate of maternal and fetal/neonatal complications was high. Pregnant SLE patients need to be monitored closely especially those with uncontrolled disease during pregnancy and history of adverse pregnancy outcome.

\section{SAFETY AND EFFICACY OF MODIFIED-RELEASE PREDNISONE IN SYSTEMIC LUPUS ERYTHEMATOUS PREGNANCIES: AN IMPLEMENTED COHORT}

${ }^{1} \mathrm{M}$ Meroni ${ }^{*},{ }^{1} \mathrm{M}$ Limonta, ${ }^{2} \mathrm{~V}$ Ramoni, ${ }^{3} \mathrm{M}$ Cutolo. 'ASST Papa Giovanni XXIII, Rheumatology, Bergamo, Italy; ${ }^{2}$ RCCS San Matteo Hospital Foundation- University of Pavia, Division of Rheumatology, Pavia, Italy; ${ }^{3}$ University of Genova- Genova-, Research Laboratory and Academic Division of Clinical Rheumatology- Department of Internal Medicineltaly, Genoa, Italy

\subsection{6/lupus-2017-000215.392}

Background and aims SLE often affects women of childbearing age. Prednisone is safely used during pregnancy. Modifiedrelease prednisone $(\mathrm{MRP})$ is a treatment option, but no data exist regarding gestation: we aimed to compare its safety and effectiveness to the immediate release formulation (IRP) in SLE pregnancies.

Methods During a 5 year period, we enrolled 18 SLE female patients experiencing a pregnancy. Nine (cases), taking lowdose MRP (5 to $7.5 \mathrm{mg} /$ daily) as a baseline treatment, were matched to 9 controls (same age and disease duration), taking the same prednisone dose in the IR formulation. Pregnancy outcome; SLEDAI/SLEPDAI; patient's VAS and need of treatment changes were assessed at baseline, during pregnancy and at postpartum.

Results SLEDAI at baseline was $2 \pm 0.1$ among MPR and 2 \pm 0.3 among IR women; SLEPDAI, $3 \pm 0.9$ and $3 \pm 0.2$ (both, $\mathrm{p}=\mathrm{ns})$. No major perinatal complications were detected. Preterm births, caesarean section rates, newborns weight and APGAR scores did not differ between the two subpopulations (all, $\mathrm{p}=\mathrm{ns}$ ). SLEDAI at postpartum was $3.8 \pm 0.6$ in MRP subjects and $5.4 \pm 0.4$ in IR $(\mathrm{p}<0.05)$. Patients VAS evaluation (MRP vs IR) was, respectively, $30 \pm 4$ and $20 \pm 9$ at baseline $(\mathrm{p}=\mathrm{ns}) ; 30 \pm 6$ and $48 \pm 7$ during pregnancy $(\mathrm{p}<0.05)$ and 31 \pm 3 and $52 \pm 9$ at postpartum $(\mathrm{p}<0.05)$. Prednisone regimen changes (add-on strategy), the observed rates involved 1/9 (MRP) and 5/9 (IR) women $(\mathrm{p}<0.05)$.

Conclusions Among IR patients, activity was significantly higher during postpartum and treatment had to be increased. VAS was significantly different (higher among IR), both during pregnancy and postpartum. MRP seems to be as safe, but more effective, in comparison the IR, during pregnancy of SLE women.

\section{PREGNANCY ASSOCIATED WITH SYSTEMIC LUPUS ERYTHEMATOSUS IN OUR HOSPITAL}

${ }^{1} \mathrm{M}$ Mukai ${ }^{*},{ }^{1} \mathrm{M}$ Kondo, ${ }^{1} \mathrm{H}$ Kanaoka, ${ }^{2} \mathrm{~K}$ Okukyama. 'Sapporo City General Hospital, Division of Rheumatology and Clinical Immunology- Department of Medicine, Sapporo, Japan; ${ }^{2}$ Sapporo City General Hospital, Department of Obstetrics, Sapporo, Japan

\subsection{6/lupus-2017-000215.393}

Background and aims To evaluate the course of pregnancy associated with systemic lupus erythematosus (SLE).

Methods We picked up retrospectively the patients with SLE who was in pregnant. We studied the courses of pregnancy, the results of delivery, and the states of babies.

Results We found 35 courses of pregnancy associated with SLE. Twenty-six patients with SLE experienced pregnancy. Thirty-six babies were born through 35 courses of pregnancy (including a pair of twin). Early deliveries were found in 5 cases (including a pair of twin). Low weight born were found 